\title{
DYNAMIC ANALYSIS OF BEAMS ON ELASTIC FOUNDATION USING FOURIER SINE TRANSFORM METHOD
}

\author{
Michael. E. Onyia and Sefa. J. Kwaghvihi \\ Department of Civil Engineering, University of Nigeria, Nsukka. Nigeria.
}

\begin{abstract}
The dynamic response of a simply supported beam resting on elastic foundation subjected to a moving uniformly distributed load is investigated in this study. The Winkler foundation model was considered and the analytical solution of the fourth order partial differential equation was obtained using the Fourier Sine Transformation Method. Numerical results were further obtained and presented in plotted curves and tabular forms. The results showed that increase in the dynamic load length, moving at constant speed leads to higher deflections and bending moments of the beam, and decreases with increasing foundation modulusk. The results thus underscore the relevance of the method in the dynamic analysis of beams on elastic foundation.
\end{abstract}

Key words: Dynamic Response, Beam, Elastic Foundation, Fourier Sine Transformation Method, Moving Uniformly Distributed Load. .

Cite this Article: Michael. E. Onyia and Sefa. J. Kwaghvihi, Dynamic Analysis of Beams on Elastic Foundation Using Fourier Sine Transform Method, International Journal of Civil Engineering and Technology, 11(04), 2020, pp.1-13

https://iaeme.com/Home/issue/IJCIET?Volume=11\&Issue $=4$

\section{INTRODUCTION}

Beams on elastic foundation have received much attention over the years due to its wide applications in engineering. Many practical engineering applications such as rail road tracks, highway pavements, buried pipelines and foundation beams among other applications, are modeled as beams resting on elastic foundation (Abohadima and Taha, 2009).Other applications include naval structures such as network of beams in the construction of floor systems for ships, earthquake-resisting structures, bridges, submerged floating tunnels, launchers, vibrating machines on elastic foundations, shafts supported on ball, etc.

Due to the wide application of this subject matter, the dynamic behaviour of structures like beams resting on elastic foundation under the influence of moving loads has attracted a lot of attention by researchers in engineering, applied physics and applied mathematics. Uzzal, et al (2012), considered the dynamic response of a beam supported by Pasternak 
foundation subjectedto moving point load and moving mass. Tiwari and Kuppa (2014) also studied the response of a beam on elastic foundation under static and dynamic loads using the finite element approach. Amiri and Onyango (2010) studied the response of a simply supported beam on elastic foundation carrying repeated rolling concentrated loads. Most of the researches were however based on moving dynamic point loads and masses using complex and cumbersome methods. This work focuses on uniformly distributed dynamic loads analysed using a more simplifiedmethod of Fourier Sine Transform..

\section{THE GOVERNING EQUATIONS}

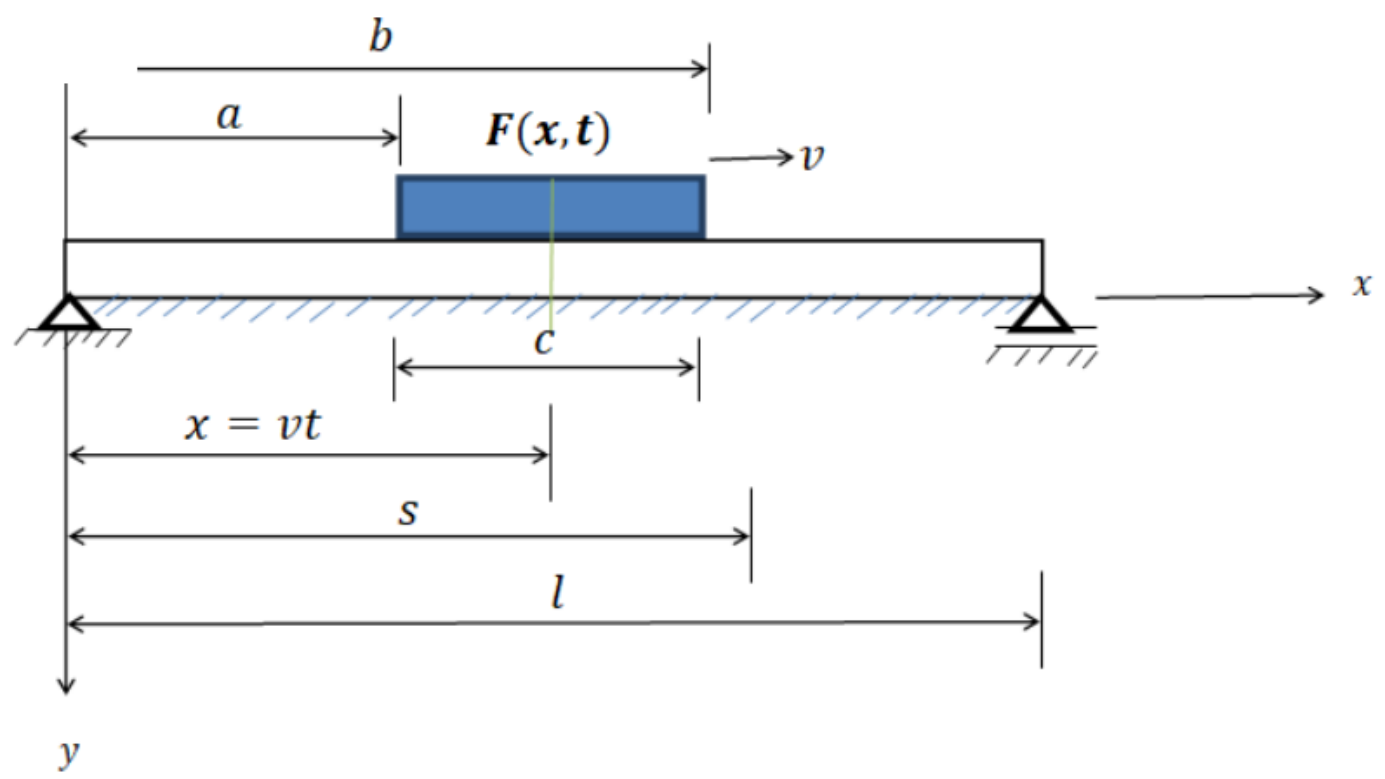

Figure 1 - A simply supported beam resting on the Winkler foundation under a moving uniformly distributed load.

The behaviour of a simply supported beam resting on Winkler foundation under the influence of uniformly distributed dynamic load (Figure 1) can be described by the fourth order differential equation:

$$
E I \frac{\partial^{4} y(x, t)}{\partial x^{4}}+m a+k y(x, t)=F(x, t)
$$

where $E I$ is the flexural rigidity of the beam, $m a$ is the force of inertia introduced into the system by the effect of the dynamic force $F(x, t), k$ is the modulus of subgrade reaction, $x$ is spatial coordinate along the beam and $t$ is time.

$$
m a=m \frac{\partial^{2} y}{\partial t^{2}}
$$

Substituting (2) in Equation (1)

$$
E I \frac{\partial^{4} y(x, t)}{\partial x^{4}}+m \frac{\partial^{2} y(x, t)}{\partial t^{2}}+k y(x, t)=F(x, t)
$$

The moving uniformly distributed load can be expressed as

$$
F(x, t)=\frac{q l}{j \pi}\left(\cos \frac{j \pi a}{l}-\cos \frac{j \pi b}{l}\right) \delta(x-v t)
$$

Substituting (4) in Equation (3) gives the governing equation as

$$
E I \frac{\partial^{4} y(x, t)}{\partial x^{4}}+m \frac{\partial^{2} y(x, t)}{\partial t^{2}}+k y(x, t)=\frac{q l}{j \pi}\left(\cos \frac{j \pi a}{l}-\cos \frac{j \pi b}{l}\right) \delta(x-v t)
$$


whereqis the magnitude of the moving uniformly distributed load, $a$ and $b$ represent the start and end points of the moving uniformly distributed load from the left support respectively and $l$ is the length of the beam.

\section{SOLUTION OF THE GOVERNING EQUATION}

Consider the beam in Figure 1subjected to the action of a moving uniformly distributed load $F(x, t)$ from the left end of the beam.

At time $t$, the differential equation for the moving uniformly distributed load $q$ is:

$$
E I \frac{\partial^{4} y(x, t)}{\partial x^{4}}+m \frac{\partial^{2} y(x, t)}{\partial t^{2}}+k y(x, t)=\frac{q l}{j \pi}\left(\cos \frac{j \pi a}{l}-\cos \frac{j \pi b}{l}\right) \delta(x-v t) \quad 0 \leq t \leq \frac{l-s}{v}
$$

The response of a simply supported beam on an elastic foundation to a moving concentrated load is given by (Amiri et al 2010):

$$
y(x, t)=\frac{P_{0}}{2 \pi^{2} m l} \sum_{j=1}^{\infty}\left[\frac{\sin 2 \pi j f t-\left(\frac{j f}{f_{j}}\right) \sin 2 \pi f t}{f_{j}^{2}-(j f)^{2}}\right] \sin \frac{j \pi x}{l}
$$

where, $P_{0}$ is magnitude of the dynamic point load, $m$ is mass of the beam per unit length, $l$ is length of the beam, $t$ is time, $f$ is fundamental forcing frequency of the dynamic load, $f_{j}$ is fundamental frequency of the beam, $j$ is index and $x$ is spatial coordinate along the beam length.

For the uniformly distributed load, the deflection curve can be obtained by putting $q d c$ instead of $P_{0}$ and by integrating between the limits $a$ and $b$ (Hetenyi, 1971).

Thus,

$q \int_{a}^{b} \sin \frac{j \pi c}{l} d c=\frac{q l}{j \pi}\left(\cos \frac{j \pi a}{l}-\cos \frac{j \pi b}{l}\right)$

where $c$ is the load length.

Hence, $F(x, t)$ can be expressed as

$$
F(x, t)=\frac{q l}{j \pi}\left(\cos \frac{j \pi a}{l}-\cos \frac{j \pi b}{l}\right) \delta(x-v t)
$$

Substituting (8) for $P_{0}$ in Equation (7) gives

$$
y(x, t)=\frac{q}{2 \pi^{3} m} \sum_{j=1}^{\infty} \frac{1}{j}\left(\cos \frac{j \pi a}{l}-\cos \frac{j \pi b}{l}\right)\left[\frac{\sin 2 \pi j f t-\left(\frac{j f}{f_{j}}\right) \sin 2 \pi f_{j} t}{f_{j}{ }^{2}-(j f)^{2}}\right] \sin \frac{j \pi x}{l}
$$

The time rate of change of Equation (10) can be expressed as

$$
\frac{\delta y(x, t)}{\delta t}=\frac{q}{2 \pi^{3} m} \sum_{j=1}^{\infty} \frac{1}{j}\left(\cos \frac{j \pi a}{l}-\cos \frac{j \pi b}{l}\right)\left[\frac{2 \pi j f\left(\cos 2 \pi j f t-\cos 2 \pi f_{j} t\right)}{f_{j}^{2}-(j f)^{2}}\right] \sin \frac{j \pi x}{l}
$$

The boundary conditions are

$$
\begin{gathered}
y(0, t)=y(l, t)=0 \\
\frac{\partial^{2} y(0, t)}{\partial t^{2}}=\frac{\partial^{2} y(l, t)}{\partial t^{2}}=0
\end{gathered}
$$

When the load is at distance $s$ from the left end of the beam, that is at time $t=\frac{s}{v}$, Equations (10) and (11) become 


$$
y\left(x, \frac{s}{v}\right)=\frac{q}{2 \pi^{3} m} \sum_{j=1}^{\infty} \frac{1}{j}\left(\cos \frac{j \pi a}{l}-\cos \frac{j \pi b}{l}\right)\left[\frac{\sin 2 \pi j f_{\frac{s}{v}}-\left(\frac{j f}{f_{j}}\right) \sin 2 \pi f_{j v}^{s}}{f_{j}^{2}-(j f)^{2}}\right] \sin \frac{j \pi x}{l}
$$

and

$$
\left.\frac{\delta y(x, t)}{\delta t}\right|_{t=\frac{s}{v}}=\frac{q}{2 \pi^{3} m} \sum_{j=1}^{\infty} \frac{1}{j}\left(\cos \frac{j \pi a}{l}-\cos \frac{j \pi b}{l}\right)\left[\frac{2 \pi j f\left(\cos 2 \pi j f_{v}^{s}-\cos 2 \pi f_{j}^{-\frac{s}{v}}\right)}{f_{j}{ }^{2}-(j f)^{2}}\right] \sin \frac{j \pi x}{l}
$$

The initial conditions are thus

$$
\begin{aligned}
& y(x, 0)=\frac{q}{2 \pi^{3} m} \sum_{j=1}^{\infty} \frac{1}{j}\left(\cos \frac{j \pi a}{l}-\cos \frac{j \pi b}{l}\right)\left[\frac{\sin 2 \pi j f_{\frac{v}{v}}^{s}-\left(\frac{j f}{f_{j}}\right) \sin 2 \pi f_{j \frac{j}{v}}}{f_{j}^{2}-(j f)^{2}}\right] \sin \frac{j \pi x}{l} \\
& \left.\frac{\delta y(x, t)}{\delta t}\right|_{t=0}=\frac{q}{2 \pi^{3} m} \sum_{j=1}^{\infty} \frac{1}{j}\left(\cos \frac{j \pi a}{l}-\cos \frac{j \pi b}{l}\right)\left[\frac{2 \pi j f\left(\cos 2 \pi j f_{v}-\cos 2 \pi f_{j} \frac{s}{j_{v}}\right)}{f_{j}{ }^{2}-(j f)^{2}}\right] \sin \frac{j \pi x}{l}
\end{aligned}
$$

Having obtained the boundary and initial conditions, Equation (6) can now be solved using method of Finite Fourier Transformation, since the system is simply supported at edges $x=0$ and $x=l$.

$$
\begin{aligned}
& Y(j, t) \\
& =\int_{0}^{l} y(x, t) \sin \frac{j \pi x}{l} d x \\
& y(x, t) \\
& =\frac{2}{l} \sum_{j=1}^{\infty} Y(j, t) \sin \frac{j \pi x}{l}
\end{aligned}
$$

$Y(j, t)$ is the transform of $y(x, t)$

Multiplying Equation (6) by $\sin \frac{j \pi x}{l}$

$$
\left[E I \frac{\partial^{4} y(x, t)}{\partial x^{4}}+m \frac{\partial^{2} y(x, t)}{\partial t^{2}}+k y(x, t)\right] \sin \frac{j \pi x}{l}=\left[\frac{q l}{j \pi}\left(\cos \frac{j \pi a}{l}-\cos \frac{j \pi b}{l}\right) \delta(x-v t)\right] \sin \frac{j \pi x}{l}
$$

Integrating Equation (20) with respect to $x$ between 0 and $l$ and considering the boundary conditions (12) and (13) yields

$$
E I \frac{\pi^{4} j^{4}}{l^{4}} Y(j, t)+m \frac{d^{2} Y(j, t)}{d t^{2}}+k Y(j, t)=\frac{q l}{j \pi}\left(\cos \frac{j \pi a}{l}-\cos \frac{j \pi b}{l}\right) \sin \frac{j \pi v t}{l}
$$

From Equation (21), the natural circular frequency of the $j^{\text {th }}$ mode of vibration of the beam can be expressed as

$$
\omega^{2}=\frac{E I \pi^{4} j^{4}+k l^{4}}{l^{4} m}
$$

The corresponding natural frequency is

$f_{j}=\frac{\omega}{2 \pi}$

While the forcing frequency is

$$
\theta=\frac{j \pi v}{l}
$$

Introducing the natural and forcing frequencies (22) and (24) into Equation (21) gives 


$$
\frac{d^{2} Y(j, t)}{d t^{2}}+\omega^{2} Y(j, t)=\frac{q l}{j \pi m}\left(\cos \frac{j \pi a}{l}-\cos \frac{j \pi b}{l}\right) \sin \theta t
$$

The general solution of Equation (25) is given as

$$
Y(j, t)=Y_{H}(j, t)+Y_{P}(j, t)
$$

where $Y_{H}(j, t)$ represents the Homogeneous solution while $Y_{P}(j, t)$ is the Particular solution.

The homogeneous solution is sought in the form

$Y_{H}(j, t)=A \sin \omega t+B \cos \omega t$

and the particular solution is sought in the form

$Y_{P}(j, t)=C \sin \theta t+D \cos \theta t$

Therefore, the particular solution is

$$
Y_{P}(j, t)=\frac{q l\left(\cos \frac{j \pi a}{l}-\cos \frac{j \pi b}{l}\right)}{j \pi m\left(\omega^{2}-\theta^{2}\right)} \sin \theta t \quad j=1,2,3,
$$

and the general solution becomes

$$
Y(j, t)=A \sin \omega t+B \cos \omega t+\frac{q l\left(\cos \frac{j \pi a}{l}-\cos \frac{j \pi b}{l}\right)}{j \pi m\left(\omega^{2}-\theta^{2}\right)} \sin \theta t
$$

Hence, the inverse transformation of Equation (30) is expressed as

$$
y(x, t)=\frac{2}{l} \sum_{j=1}^{\infty}\left[A \sin \omega t+B \cos \omega t+\frac{q l\left(\cos \frac{j \pi a}{l}-\cos \frac{j \pi b}{l}\right)}{j \pi m\left(\omega^{2}-\theta^{2}\right)} \sin \theta t\right] \sin \frac{j \pi x}{l}
$$

The constants $\mathrm{A}$ and $\mathrm{B}$ in Equation (31) can be determined by applying the initial conditions (16) and (17) respectively.

From Equation (31)

$$
\left.y(x, t)\right|_{t=0}=\frac{2}{l} \sum_{j=1}^{\infty} B \sin \frac{j \pi x}{l}
$$

and

$$
\left.\frac{\partial y(x, t)}{\partial t}\right|_{t=0}=\frac{2}{l} \sum_{j=1}^{\infty}\left[A \omega+\frac{\theta q l\left(\cos \frac{j \pi a}{l}-\cos \frac{j \pi b}{l}\right)}{j \pi m\left(\omega^{2}-\theta^{2}\right)}\right] \sin \frac{j \pi x}{l}
$$

The initial conditions (16) and (17) can also be expressed in the form

$$
\left.y(x, t)\right|_{t=0}=\frac{q}{\pi^{3} m} \sum_{j=1}^{\infty} \frac{1}{j}\left(\cos \frac{j \pi a}{l}-\cos \frac{j \pi b}{l}\right)\left[\frac{\sin \theta \frac{s}{v}-\left(\frac{\theta}{\omega}\right) \sin \omega \frac{s}{v}}{\omega^{2}-\theta^{2}}\right] \sin \frac{j \pi x}{l}
$$

and

$$
\left.\frac{\partial y(x, t)}{\partial t}\right|_{t=0}=\frac{q}{\pi^{3} m} \sum_{j=1}^{\infty} \frac{1}{j}\left(\cos \frac{j \pi a}{l}-\cos \frac{j \pi b}{l}\right)\left[\frac{\theta\left(\cos \theta_{v}^{s}-\cos \omega_{v}^{s}\right)}{\omega^{2}-\theta^{2}}\right] \sin \frac{j \pi x}{l}
$$

Equating Equations (32) and (34) gives

$$
B=\frac{2 q l\left(\cos \frac{j \pi a}{l}-\cos \frac{j \pi b}{l}\right)}{j \pi m\left(\omega^{2}-\theta^{2}\right)}\left[\sin \theta \frac{s}{v}-\left(\frac{\theta}{\omega}\right) \sin \omega \frac{s}{v}\right]
$$

Similarly, equating Equations (33) and (35) gives

$$
A=\frac{q l\left(\cos \frac{j \pi a}{l}-\cos \frac{j \pi b}{l}\right)\left(\frac{\theta}{\omega}\right)}{j \pi m\left(\omega^{2}-\theta^{2}\right)}\left[2\left(\cos \theta \frac{s}{v}-\cos \omega \frac{s}{v}\right)-1\right]
$$


Substituting (36) and (37) for the constants into (31) and simplifying, gives the closed form response of the beam to the moving uniformly distributed load as

$$
y(x, t)=\frac{2 q}{\pi m} \sum_{j=1}^{\infty} \frac{\left(\cos \frac{j \pi a}{l}-\cos \frac{j \pi b}{l}\right)}{j\left(\omega^{2}-\theta^{2}\right)}\left[2 \sin \theta t-\left(\frac{\theta}{\omega}\right) \sin \omega t\right] \sin \frac{j \pi x}{l}
$$

Therefore, substituting for $\omega$ and $\theta$ in (38) gives the response as,

$$
y(x, t)=\frac{q}{2 \pi^{3} m} \sum_{j=1}^{\infty} \frac{\left(\cos \frac{j \pi a}{l}-\cos \frac{j \pi b}{l}\right)}{j\left(f_{j}{ }^{2}-j f^{2}\right)}\left[2 \sin 2 \pi j f t-\left(\frac{j f}{f_{j}}\right) \sin 2 \pi f_{j} t\right]
$$

Equation (39) is the closed form solution of the governing equation of the dynamic response of beam on Winkler foundation with simply supported ends under the influence of a moving uniformly distributed load.

The corresponding static solution is obtained by substituting $v=0$ and $t=\frac{x}{l}$ into Equation (39) which gives

$$
y_{s t}=\frac{2 q l^{4}}{\pi E I} \sum_{j=1}^{\infty} \frac{\left(\cos \frac{j \pi a}{l}-\cos \frac{j \pi b}{l}\right) \sin \frac{j \pi x}{l}}{j\left[(j \pi)^{4}+\frac{k l^{4}}{E I}\right]}
$$

Expressions for the bending moments can also be obtained from the resulting expression for the deflection in Equation (39).

$$
-M(x, t)=E I \frac{d^{2} y(x, t)}{d x^{2}}
$$

where $M$ is the Bending Moment, $E I$ is the flexural rigidity of the beam.

Therefore, at any time $t=\frac{s}{v}$ on the beam, expression for the bending moment is obtained from the second derivative of Equation (39) as,

$$
M(x, t)=\frac{E I q}{2 \pi m l^{2}} \sum_{j=1}^{\infty} \frac{j\left(\cos \frac{j \pi a}{l}-\cos \frac{j \pi b}{l}\right)}{\left(f_{j}{ }^{2}-j f^{2}\right)}\left[2 \sin 2 \pi j f t-\left(\frac{j f}{f_{j}}\right) \sin 2 \pi f_{j} t\right] \sin \frac{j \pi x}{l}
$$

\section{NUMERICAL EXAMPLE AND DISCUSSIONS}

The response of the beam in terms of deflection andbending moment at any point of distance $x$ along the beam with respect to the dynamic load location at the middle of the beam will be obtained with varying load length and the Winkler foundation constant respectively.

Consider the simply supported beam of length $l=10 \mathrm{~m}$, under the action of a dynamic uniformly distributed load of amplitude $q=200 \times 10^{3} \mathrm{~N} / \mathrm{m}$, beam modulus of elasticity $E=$ $3.0 \times 10^{10} \mathrm{~N} / \mathrm{m}^{2}$, beam moment of inertia $I=1.042 \times 10^{-2} \mathrm{~m}^{4}$, mass per unit length, $m=$ $1223.242 \mathrm{~kg} / \mathrm{m}$, the elastic foundation (Winkler) constant $k=50 \times 10^{6} \mathrm{~N} / \mathrm{m}^{2}$ and velocity of the moving load $v=10 \mathrm{~m} / \mathrm{s} . j=1$

From the above data, the flexural rigidity of the beam can be evaluated.

That is; $E I=3.0 \times 10^{10} \times 1.042 \times 10^{-2}=3.126 \times 10^{8} \mathrm{Nm}^{2}$

The natural frequency can be obtained from Equations (22) and (23) as,

$$
\omega^{2}=\frac{E I \pi^{4} j^{4}+k l^{4}}{l^{4} m}=\frac{\left(3.126 \times 10^{8} \times \pi^{4} \times 1\right)+\left(50 \times 10^{6} \times 10^{4}\right)}{1223.242 \times 10^{4}}
$$

This gives, $\omega=208.241 s^{-1}$

The natural frequency is 
Dynamic Analysis of Beams on Elastic Foundation Using Fourier Sine Transform Method

$$
f_{j}=\frac{\omega}{2 \pi}=\frac{208.241}{2 \pi}=33.143 s^{-1}
$$

While the forcing frequency can be obtained from Equation (24) as;

$$
\begin{gathered}
\theta=\frac{j \pi v}{l}=\frac{1 \times 10 \times \pi}{10}=3.142 \mathrm{~s}^{-1} \\
f=\frac{\theta}{2 \pi}=\frac{3.142}{2 \pi}=0.5 \mathrm{~s}^{-1}
\end{gathered}
$$

If the load is moving at a constant speed of $10 \mathrm{~m} / \mathrm{s}$, at mid-point of the beam(where the effect of the load is assumed to be maximum for a simply supported beam) and the distance $s=5 \mathrm{~m}$, time $t=0.5 \mathrm{sec}$., load length of $2 \mathrm{~m}, a=4 \mathrm{~m}$ and $b=6 \mathrm{~m}$ from the left end of the beam:

Recall;

$$
y(x, t)=\frac{q}{2 \pi^{3} m} \sum_{j=1}^{\infty} \frac{\left(\cos \frac{j \pi a}{l}-\cos \frac{j \pi b}{l}\right)}{j\left(f_{j}^{2}-j f^{2}\right)}\left[2 \sin 2 \pi j f t-\left(\frac{j f}{f_{j}}\right) \sin 2 \pi f_{j} t\right] \sin \frac{j \pi x}{l}
$$

Substituting the given values in Equation (39) as stated above.

$$
\begin{aligned}
& y(x, t)=\frac{200 \times 10^{3}\left(\cos \frac{4 \pi}{10}-\cos \frac{6 \pi}{10}\right)}{2 \pi^{3} \times 1223.242 \times 1\left(33.143^{2}-0.5^{2}\right)}[2 \sin 2 \pi(0.5)(0.5) \\
& \left.-\left(\frac{0.5}{33.143}\right) \sin 2 \pi(33.143)(0.5)\right] \sin \frac{\pi x}{10}
\end{aligned}
$$

This gives the dynamic response at any point $x$ on the beam as

$$
y(x, t)=0.002977 \sin \frac{\pi x}{10}
$$

The mid-point deflection values of the beam are obtained and presented in Table 4.0 below for different values of the foundation constant $k$, when the load length is $2 \mathrm{~m}$ and located at the center of the beam.

The corresponding static response can be obtained by substituting the respective values of the parameters in Equation (40) above, which is expressed as

$$
y_{s t}=\frac{2 q l^{4}}{\pi E I} \sum_{j=1}^{\infty} \frac{\left(\cos \frac{j \pi a}{l}-\cos \frac{j \pi b}{l}\right) \sin \frac{j \pi x}{l}}{j\left[(j \pi)^{4}+\frac{k l^{4}}{E I}\right]}
$$

Substituting the given numerical values in the above expression for the static response gives,

$$
y_{s t}=\frac{2 \times 200 \times 10^{3} \times 10^{4}\left(\cos \frac{4 \pi}{10}-\cos \frac{6 \pi}{10}\right)}{\pi \times 312.6 \times 10^{6} \times 1\left[\pi^{4}+\left(\frac{50 \times 10^{6} \times 10^{4}}{312.6 \times 10^{6}}\right)\right]} \sin \frac{\pi x}{10}
$$

This gives the static response at any point $x$ on the beam as

$$
y_{s t}=0.001483 \sin \frac{\pi x}{10}
$$


Hence, the corresponding static deflection values at mid-point of the beam are obtained and presented in Table 4.0 below, for different values of the foundation constant $k$, when the load length is $2 \mathrm{~m}$ and located at the center of the beam.

The obtained values of the static deflection from this approach are compared to the values obtained from Hetenyi's Trigonometric Series method, which gives the deflection line of a beam on elastic foundation under the influence of a static uniformly distributed load as: (Hetenyi, 1971)

$$
y=\frac{2 q l^{4}}{\pi^{5} E I} \sum_{n=1}^{\infty} \frac{\left(\cos \frac{n \pi c_{1}}{l}-\cos \frac{n \pi c_{2}}{l}\right) \sin \frac{n \pi x}{l}}{n\left(n^{4}+\frac{k l^{4}}{\pi^{4} E I}\right)}
$$

where $q$ is the uniformly distributed load, $c_{1}$ and $c_{2}$ are the start and end points of the load from the left support of the beam respectively, $E I$ is the flexural rigidity of the beam, $k$ is the foundation constant, $l$ is the beam length and $n$ is the index, $1,2,3, \ldots$

In this case, the value of the uniformly distributed load $q=200 \times 10^{3} \mathrm{~N} / \mathrm{m}$, the load start point from the left end of the beam $c_{1}=4 \mathrm{~m}$, while its endpoint from the left of the beam $c_{2}=6 \mathrm{~m}$, modulus of elasticity of the beam $E=3.0 \times 10^{10} \mathrm{~N} / \mathrm{m}^{2}$, moment of inertia $I=1.042 \times 10^{-2} \mathrm{~m}^{4}$, the foundation constant $k=50 \times 10^{6} \mathrm{~N} / \mathrm{m}^{2}$, the length of the beam $l=10 \mathrm{~m}$ and the index $n=1$

Therefore, substituting the above values in Equation (43) gives the corresponding static deflection values at mid-point of the beam using Hetenyi's Trigonometric Series method and presented in Table I below, for different values of the foundation constant $k$, when the load length is $2 \mathrm{~m}$ and located at the center of the beam.

TABLE I Mid-Point Deflections for Different K-Values and the Dynamic Amplification Factors

\begin{tabular}{|c|c|c|c|c|}
\hline & $\begin{array}{c}\text { MID-POINT } \\
\text { DYNAMIC } \\
\text { RESPONSE OF THE } \\
\text { BEAM }\end{array}$ & $\begin{array}{c}\text { CORRESPONDING } \\
\text { MID-POINT } \\
\text { STATIC BEAM } \\
\text { RESPONSE }\end{array}$ & $\begin{array}{c}\text { HETENYI'S } \\
\text { MID-POINT } \\
\text { STATIC } \\
\text { DEFLECTION }\end{array}$ & $\begin{array}{c}\text { DYNAMIC } \\
\text { AMPLIFICATION } \\
\text { FACTOR }\end{array}$ \\
\hline 0 & $\boldsymbol{y}(\boldsymbol{x}, \boldsymbol{t})$ & $\boldsymbol{y}_{\boldsymbol{s} \boldsymbol{t}}$ & $\boldsymbol{y}(\boldsymbol{x}, \boldsymbol{t})$ & $\boldsymbol{\mu}=\frac{\boldsymbol{y}_{\boldsymbol{s} \boldsymbol{t}}}{}$ \\
\hline $5 \times 10^{6}$ & 0.052178768 & 0.025837224 & 0.025837224 & 2.019519 \\
\hline $10 \times 10^{6}$ & 0.011895041 & 0.006031927 & 0.006031927 & 1.972013 \\
\hline $15 \times 10^{6}$ & 0.008824399 & 0.004360627 & 0.004360627 & 2.023654 \\
\hline $20 \times 10^{6}$ & 0.006869189 & 0.003414541 & 0.003414541 & 2.011746 \\
\hline $25 \times 10^{6}$ & 0.005596203 & 0.002805793 & 0.002805793 & 1.994517 \\
\hline $30 \times 10^{6}$ & 0.00474245 & 0.00238126 & 0.00238126 & 1.991572 \\
\hline $35 \times 10^{6}$ & 0.004130087 & 0.002068312 & 0.002068312 & 1.996839 \\
\hline $40 \times 10^{6}$ & 0.003670563 & 0.001828065 & 0.001828065 & 2.007895 \\
\hline $45 \times 10^{6}$ & 0.003302214 & 0.001637823 & 0.001637823 & 2.016222 \\
\hline $50 \times 10^{6}$ & 0.002977271 & 0.001483444 & 0.001483444 & 2.006999 \\
\hline
\end{tabular}


From Table Iabove, there is a clear agreement between the corresponding static deflection values obtained using the Fourier Sine Transform Method and those by Hetenyi's Trigonometric Series method. This demonstrates the validity of the Fourier Sine Transform Method

Also, the dynamic deflection values are expectedly higher than the corresponding static values, giving Dynamic Amplification Factor greater than 1.0 in each case. (Ibearugbulem et. al, 2014).

\subsection{BENDING MOMENTS}

Bending moments for the beam under the influence of the dynamic uniformly distributed load can be obtained from Equation (42). Thus

$$
M(x, t)=\frac{E I q}{2 \pi m l^{2}} \sum_{j=1}^{\infty} \frac{j\left(\cos \frac{j \pi a}{l}-\cos \frac{j \pi b}{l}\right)}{\left(f_{j}^{2}-j f^{2}\right)}\left[2 \sin 2 \pi j f t-\left(\frac{j f}{f_{j}}\right) \sin 2 \pi f_{j} t\right] \sin \frac{j \pi x}{l}
$$

Substituting the given values in Section 4.1 above, gives

$$
\begin{aligned}
M(x, t) & =\frac{3.126 \times 10^{8} \times 200 \times 10^{3} \times 1\left(\cos \frac{4 \pi}{10}-\cos \frac{6 \pi}{10}\right)}{2 \pi \times 1223.242 \times 10^{2}\left(33.143^{2}-0.5^{2}\right)}[2 \sin 2 \pi(0.5)(0.5) \\
& \left.-\left(\frac{0.5}{33.143}\right) \sin 2 \pi(33.143)(0.5)\right] \sin \frac{\pi x}{10}
\end{aligned}
$$

Therefore, the bending moments at any point $x$ on the beam is

$$
M(x, t)=91850.3396 \sin \frac{\pi x}{10}
$$

A summary of the dynamic response of the beam in terms of the deflections and bending moments resulting from the Numerical Example is shown in Table II

TABLE II Dynamic Response Values from the Numerical Example

\begin{tabular}{|c|c|c|}
\hline $\boldsymbol{x}(\mathrm{m})$ & $\begin{array}{c}\text { Deflection }(\mathrm{m}) \\
\boldsymbol{y}(\boldsymbol{x}, \boldsymbol{t})\end{array}$ & $\begin{array}{c}\text { Bending Moments }(\mathrm{KNm}) \\
\boldsymbol{M}(\boldsymbol{x}, \boldsymbol{t})\end{array}$ \\
\hline 0 & 0 & 0 \\
\hline 1 & 0.000920143 & 53.99432985 \\
\hline 2 & 0.001750192 & 74.31508426 \\
\hline 3 & 0.002408877 & 87.35948941 \\
\hline 4 & 0.002831703 & 91.85033962 \\
\hline 5 & 0.002977271 & 87.35948941 \\
\hline 6 & 0.002831703 & 74.31508426 \\
\hline 7 & 0.002408877 & 53.99432985 \\
\hline 8 & 0.001750192 & 28.38687482 \\
\hline 9 & 0.000920143 & 0 \\
\hline 10 & 0 & \\
\hline
\end{tabular}


Table II shows that for the hinged beam analysed using the Fourier Sine Transform method,the moments at the supports are expectedly zero, while the maximum bending moment $(91.85 \mathrm{kNm})$ occurs at the middle of the beam (Figure 1).

\subsection{EFFECT OF LOAD LENGTH ON DYNAMIC RESPONSE OF THE BEAM}

To further investigate the relationship between the beam response and the load length, the length of the uniformly distributed load is varied between $0.1 l$ to $l$ of the beam with reference to the center for different values of the foundation constant $k$. The resulting relationship is presented graphically by considering load lengths of $0.1 l, 0.5 l$ and $l$ respectively as follows;

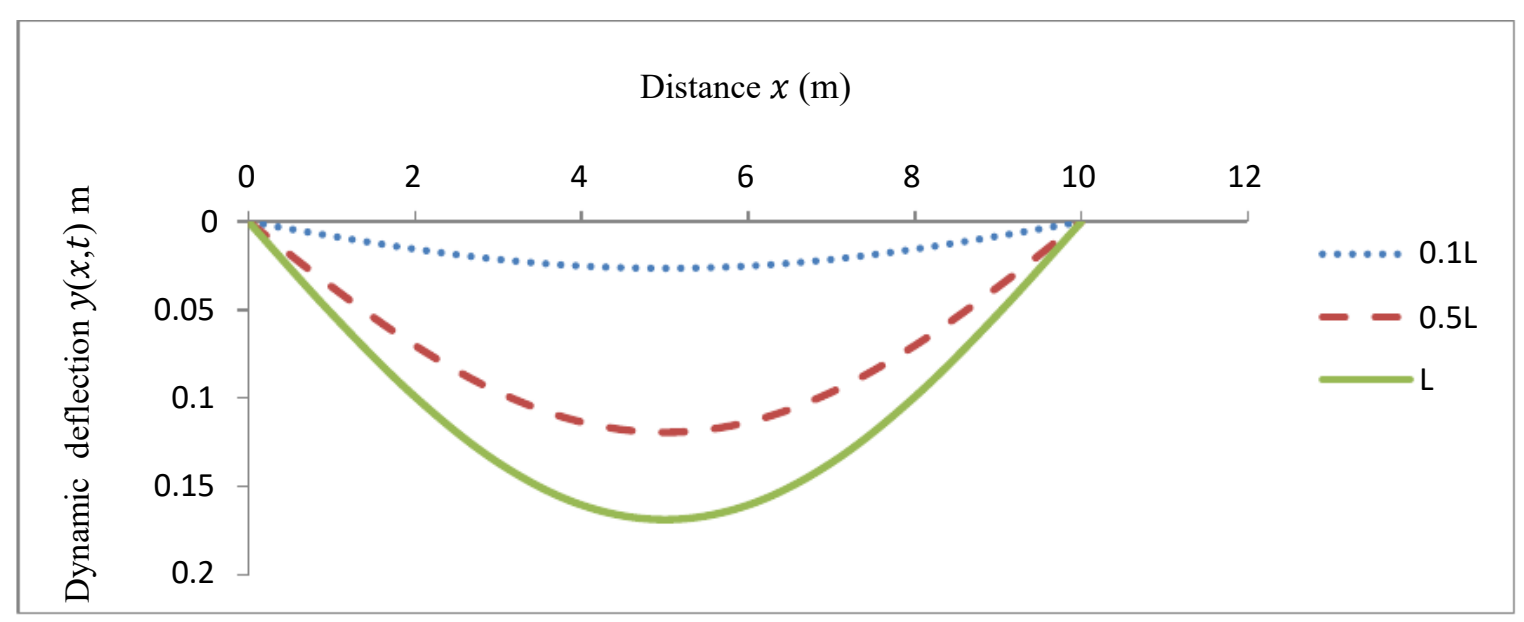

Figure. 1 Dynamic deflection of the beam under uniformly distributed load for different load lengths when $k=0$

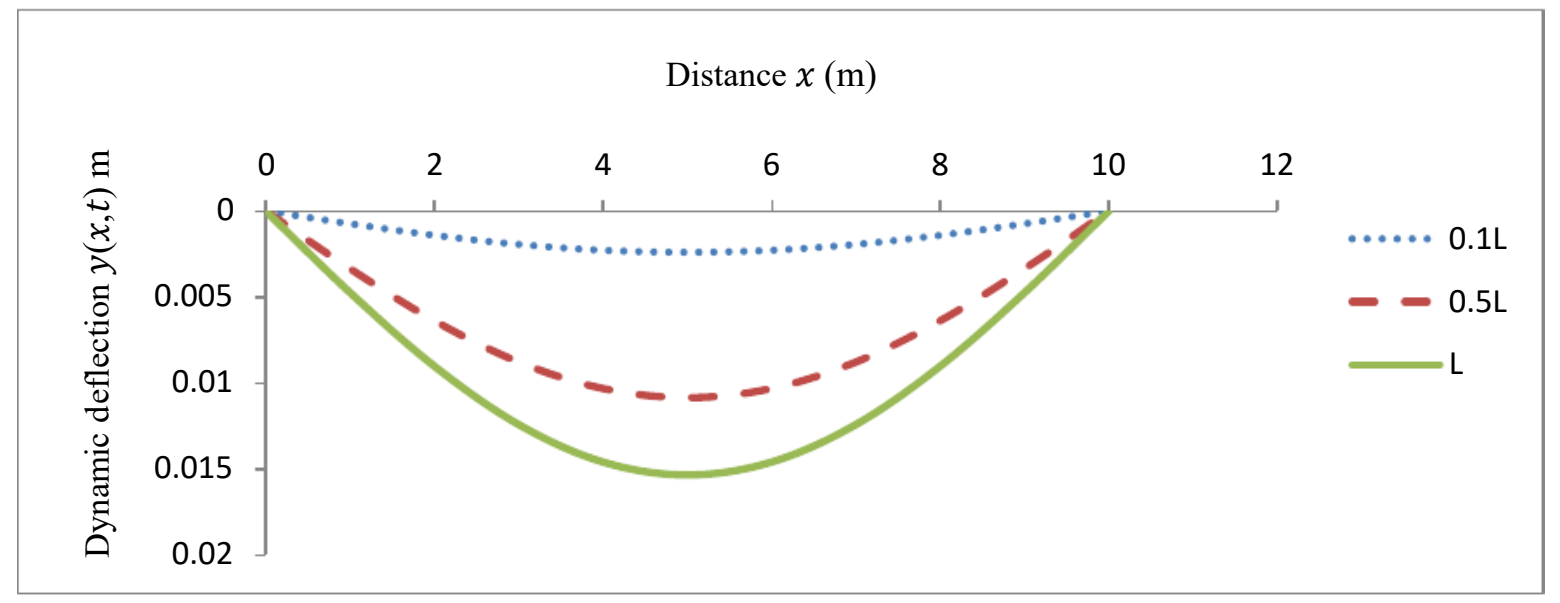

Figure. 2 Dynamic deflection of the beam under uniformly distributed load for different load lengths when $k=30 \times 10^{6} \mathrm{~N} / \mathrm{m}^{2}$ 


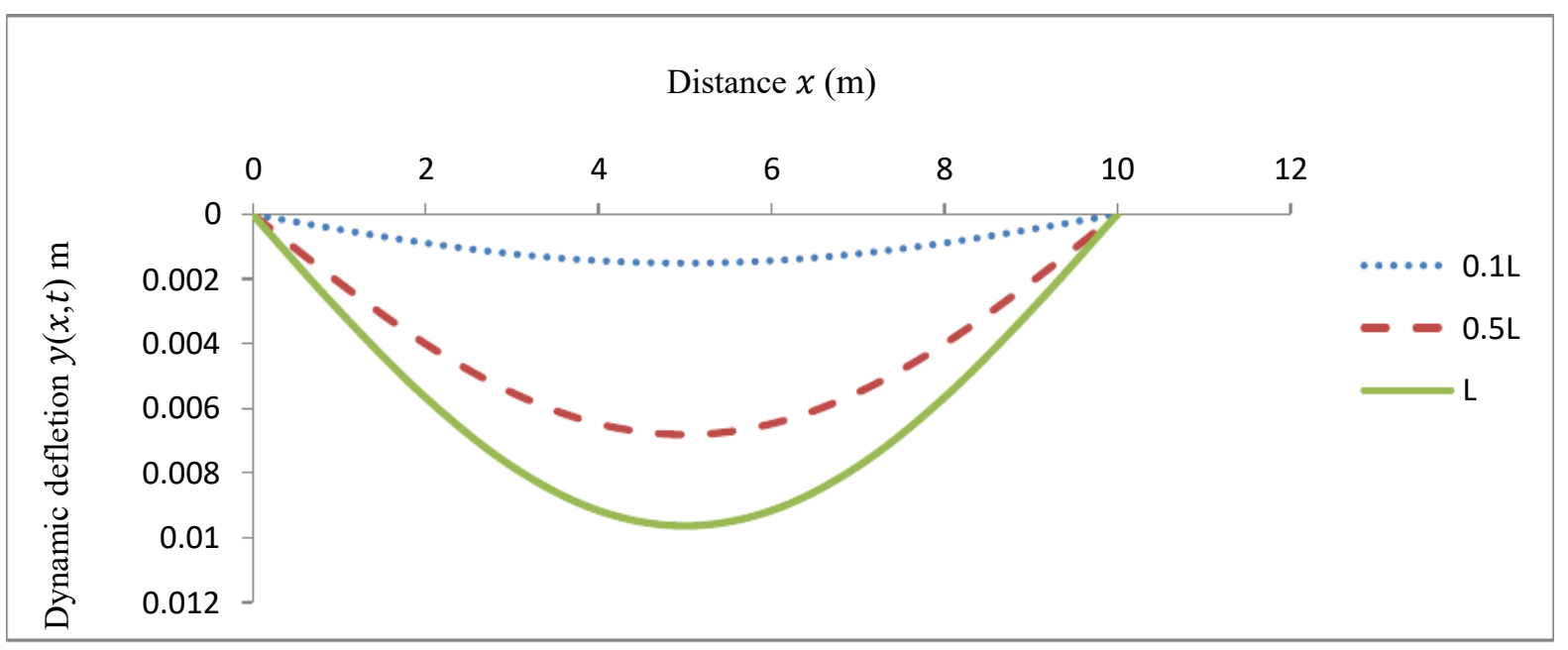

Figure. 3 Dynamic deflection of the beam under uniformly distributed load for different load lengths when $k=50 \times 10^{6} \mathrm{~N} / \mathrm{m}^{2}$

Figure 1 to Figure 3 show that the load length has a significant effect on the beam response. At constant speed, increase in the load length results in higher deflection of the beam on elastic foundation. This implies that when the load length is equal to the length of the beam, the system tends towards resonance faster, even at constant speed.

Also, at constant speedthe dynamic deflection (response) decreases as the foundation stiffness $k$ increases, and vice versa.

\subsection{BENDING MOMENT CURVES}

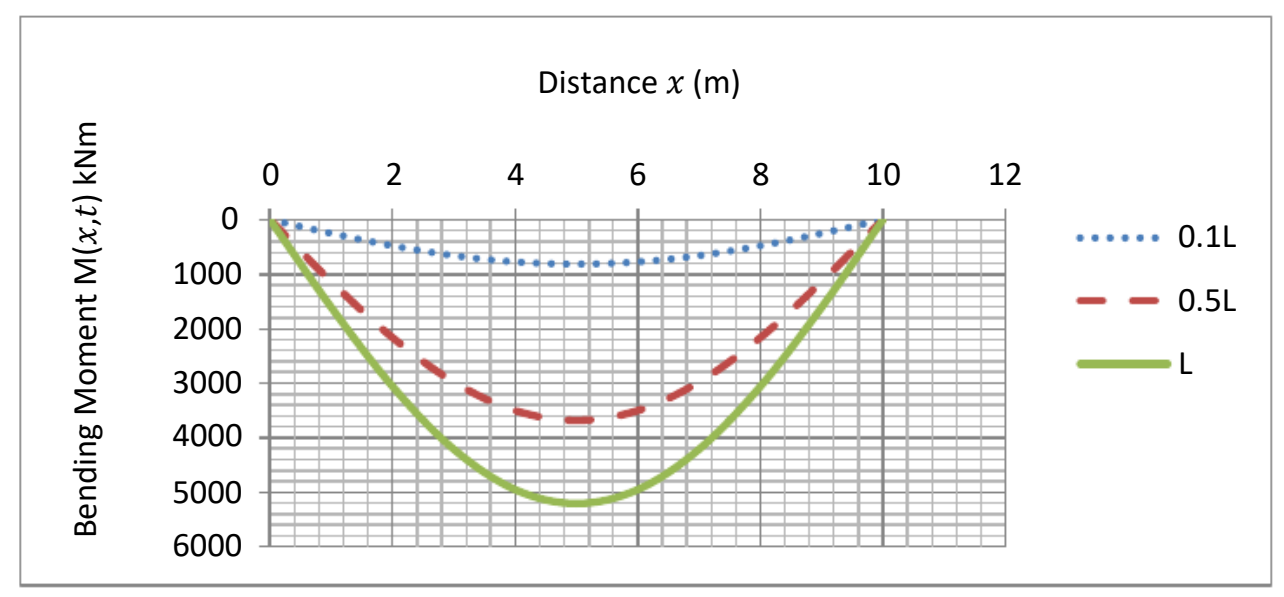

Figure. 4 Bending Moment diagram of the beam under uniformly distributed dynamic load for varying load lengths when $k=0$ 


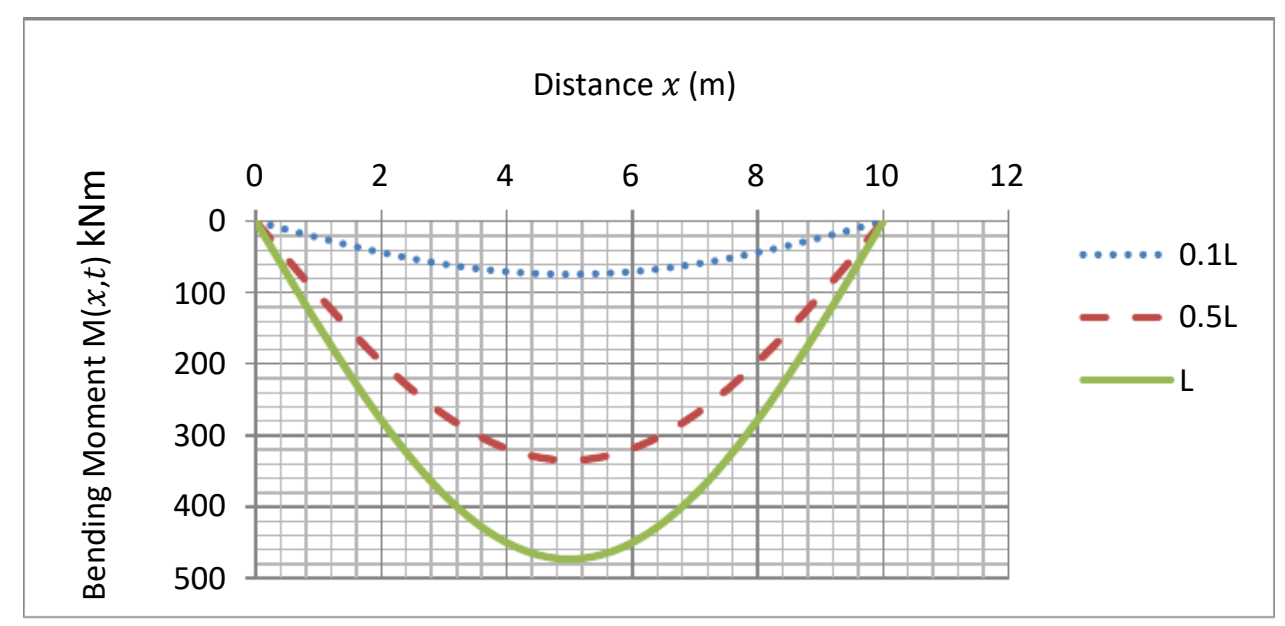

Figure. 5 Bending Moment diagram of the beam under uniformly distributed dynamic load for varying load lengths when $k=30 \times 10^{6} \mathrm{~N} / \mathrm{m}^{2}$

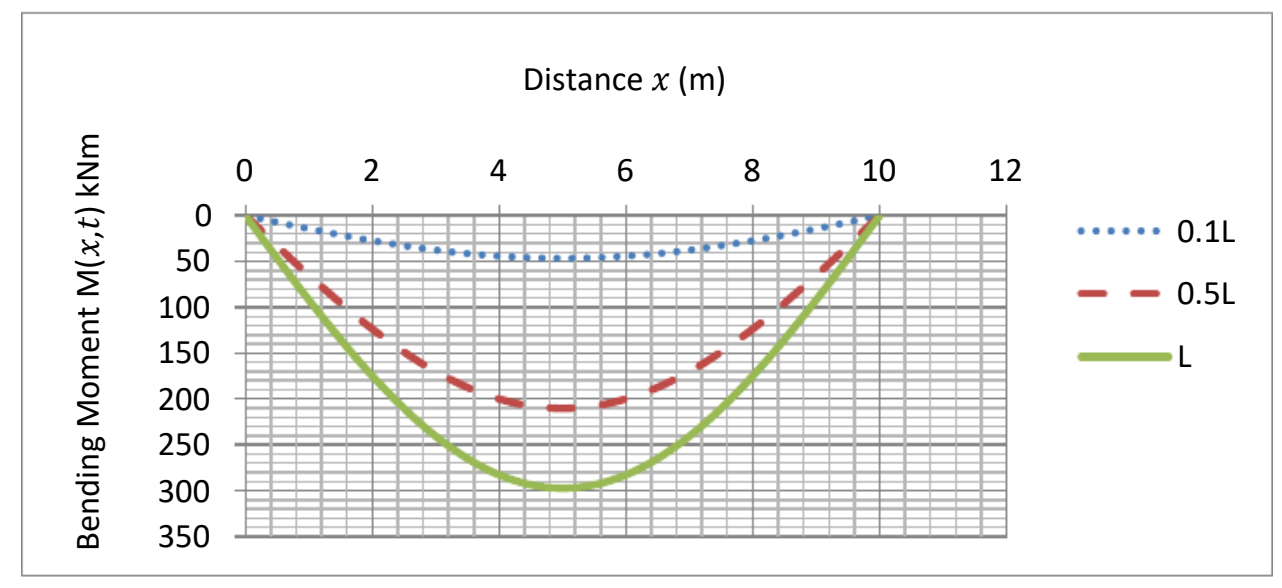

Figure. 6 Bending Moment diagram of the beam under uniformly distributed dynamic load for varying load lengths when $k=50 \times 10^{6} \mathrm{~N} / \mathrm{m}^{2}$.

Figure 3 to Figure 6 show that the foundation stiffness $k$ has significant effect on the beam bending moments. It can be deduced that at constant speed, the bending moment (response) decreases as the foundation stiffness $k$ increases, and vice versa.

Also, increase in the load length, moving at constant speed, results in higher bending moments of the beam on elastic foundation (Uzzal et. al, 2012, Oni et. al, 2010).

\section{CONCLUSION}

In this work, the dynamic response of a simply supported beam resting on elastic foundation subjected to a moving uniformly distributed load is investigated. The closed form solution of the derived governing fourth order partial differential equation was obtained by means of the Fourier Sine Transformation method. Numerical examples were solved and the results compared with previously published work by Hetenyi (1971), and were found to be in good agreement. Various analyses were also done on the dynamic system, while the response in terms of deflection andbending moments were studied.

The study demonstrates that increase in the dynamic uniformly distributed load length, moving at constant speed, leads to higher deflections and bending moments.It is therefore important for designers to take the load length into consideration to avoid the undesirable phenomenon of resonance which results from excessively large deflections. It also shows that 
the response amplitudes of the beam on elastic foundation decrease with increasing foundation modulus $(k)$.

\section{REFERENCES}

[1] Abohadima, S. and Taha, M. H. (2009):"Dynamic Analysis of Non-uniform Beams on Elastic Foundations", The Open Applied Mathematics Journal, Vol.3, pp. 40 -44.

[2] Amiri, S.N. and Onyango, M. (2010):"Simply Supported Beam Response on Elastic Foundation Carrying Repeated Rolling Concentrated Loads", Journal of Engineering Science and Technology, Vol.5, No.1, pp. 52 -66.

[3] Basu, H.E. (2017):"Dynamic Response of Simply Supported Beams on Two-Parameter Foundations", Proceedings of the 19th International Conference on Soil Mechanics and Geotechnical Engineering Seoul, pp. 729-732.

[4] Chang, S. (1965):"Infinite Beams on an Elastic Foundation”, M.Sc. Thesis submitted to the Faculty of the University of Missouri, Rolla.

[5] Dobromir, D. (2012):"Analytical Solution of Beam on Elastic Foundation by Singularity Functions”, Journal of Engineering Mechanics, Vol. 19, pp. 381-392.

[6] Elhuni, H. and Basu, D. (2017):"Dynamic Response of Simply Supported Beams on Two-parameter Foundations", Proceedings of the 19th International Conference on Soil Mechanics and Geotechnical Engineering, Seoul.

[7] Hetényi, M. (1971):"Beams on Elastic Foundation", Ninth Edition, University of Michigan Press, U.S.A.

[8] Ibearugbulem, O. M., Ezeh, J. C. and Ettu, L. O. (2014):“Energy Methods in Theory of Rectangular Plates", Liu House of Excellence Ventures Owerri, Imo State, Nigeria.

[9] Mehri, B., Davar, A. and Rahmani, O. (2009):"Dynamic Green Function Solution of Beams Under a Moving Load with Different Boundary Conditions", Journal of Mechanical Engineering, Vol. 16, No. 3, pp. 273 - 279.

[10] Ogunbamike, O. K. (2012):"Response of Timoshenko Beams on Winkler Foundation Subjected to Dynamic Load", International Journal of Scientific and Technology Research, Vol. 1, Issue 8, pp. $48-51$.

[11] Ogunyebi, S.N. (2017):"Response of Beam to Variable Elastic Subgrade Subjected to a Distributed Harmonic Loading Condition", American Journal of Innovative Research and Applied Sciences, Vol. 4, Issue 2, pp. 59-63.

[12] Ojih, P. B., Ibiejugba, M. A. and Adejo, B. O. (2014):"Dynamic Response under Moving Concentrated Loads of Uniform Rayleigh Beam Resting on Pasternak Foundation", International Journal of Science and Engineering Research, Vol. 5, Issue 6, pp. 1610 1630 .

[13] Omolofe, B. and Adeloye, T.O. (2017):"Behavioural Study of Finite Beam Resting on Elastic Foundation and Subjected to Travelling Distributed Masses", Latin American Journal of Solids and Structures, Vol. 14, pp. 312-334.

[14] Oni, S.T. and Awodola, T.O. (2010):"'Dynamic Response under a Moving Load of an Elastically Supported Non-prismatic Bernoulli-Euler Beam on Variable Elastic Foundation", Latin American Journal of Solid Structures, Vol. 7, pp. 3-20

[15] Taha, M. H. and Doha, E. H. (2015):"Recursive Differentiation Method: Application to the Analysis of Beams on Two Parameter Foundations", Journal of Theory of Applied Mechanics, Sofia, Vol. 53, No. 1, pp. 15 - 26.

[16] Tiwari, K. and Kuppa, R. (2014):"Overview of Methods of Analysis of Beams on Elastic Foundation”, Journal of Mechanical and Civil Engineering, Vol. 11, Issue 5, pp. $22-29$.

[17] Uzzal, R.U.A., Bhat, R.B. and Ahmed W. (2012):"Dynamic Response of a Beam Subjected to Moving Load and Moving Mass Supported by Pasternak Foundation", Journal of Shock and Vibration, Vol. 19, pp. $205-220$. 